\title{
The application of nutrition support in conservative treatment of chylous ascites after abdominal surgery
}

This article was published in the following Dove Press journal:

Therapeutics and Clinical Risk Management

15 April 2016

Number of times this article has been viewed

\author{
Wu Pan' \\ Shen-Yang $\mathrm{Cai}^{2}$ \\ Hai-Long Luo ${ }^{2}$ \\ Shu-Rui Ouyang' \\ Wen-Duo Zhang' \\ Zai-Rong Wei' \\ Da-Li Wang'
}

'Department of Plastic \& Vascular Surgery, Affiliated Hospital

of Zunyi Medical College,

Zunyi, Guizhou, ${ }^{2}$ Department of

Hepatopancreatobiliary Surgery,

West China Hospital, Sichuan

University, Chengdu, Sichuan,

People's Republic of China
Correspondence: Da-Li Wang

Department of Plastic \& Vascular Surgery, Affiliated Hospital of Zunyi Medical

College, I 49 Dalian Road, Zunyi 563000,

Guizhou, People's Republic of China

$\mathrm{Tel} / \mathrm{fax}+8685$ I 28609676

Email wangdalee@yeah.net
Background: Chylous ascites is the pathologic leakage of triglycerides-rich lymphatic fluid into the peritoneal cavity. Chylous ascites is a rare complication in abdominal surgery. This study aimed to find a relatively better method for nutrition support in the treatment of chylous ascites after abdominal surgery.

Methods: This study was a retrospective study. This study retrospectively reviewed patients who underwent abdominal surgery and developed chylous ascites, from the year 2010 to 2014 , at the West China Hospital of Sichuan University and the Affiliated Hospital of Zunyi Medical College. Fifty-eight patients who developed chylous ascites after abdominal surgery were included in the study. The clinical effect of somatostatin was evaluated. The differences in the curative efficacy among a daily diet, a low-fat diet supplemented with medium-chain triglyceride (MCT), and total parenteral nutrition (TPN) were also analyzed in this study.

Results: Complete clinical success was reached earlier in patients treated with somatostatin $(P<0.001)$. The tube removal time, the time to resumption of an oral diet, and the length of hospital stay after chylous leakage were significantly different between patients treated with and without somatostatin. The curative efficacies of the enteral nutrition (EN) + MCT plan and the TPN plan were quite similar, with no significant difference, however, were significantly different from the MCT regime, which was the worst. However, using the EN + MCT plan was more cost-effective ( $P=0.038$ ).

Conclusion: In treating chylous ascites, EN + MCT instead of TPN was the best nutrition support. Moreover, somatostatin or its analog octreotide should be used immediately. The treatment with somatostatin in combination with $\mathrm{EN}+\mathrm{MCT}$ is recommended in the conservative treatment of postoperative chylous ascites.

Keywords: chylous ascites, somatostatin, medium-chain triglycerides, enteral nutrition, total parenteral nutrition

\section{Introduction}

Chylous ascites is the pathologic leakage of triglycerides-rich lymphatic fluid into the peritoneal cavity. ${ }^{1,2}$ It may be produced by malignancy, surgery, radiotherapy, tuberculosis, filariasis, trauma, cirrhosis or nephrotic syndrome, and congenital abnormality of the lymphatic system. ${ }^{1}$ Chylous ascites is a rare complication in abdominal surgery $\left(\sim 1.0 \%\right.$ in hepatopancreatobiliary surgery). ${ }^{3}$ Because its volume is very large and rich in nutrients, it may lead to malnutrition, dehydration, electrolyte imbalance, and delayed wound healing. Moreover, as chylous ascites contains lymphatic fluid which is rich in lymphocytes and immunoglobulins, severe and long-term chylous leakage may cause hypoimmunity, ${ }^{1}$ therefore leading to severe infection or even death 
because of sepsis. Therefore, timely and effective treatment is necessary. Because it is a rare postoperative complication, little is known about it, and the treatment of chylous ascites usually relies on experience.

The management of chylous ascites includes surgery and conservative treatment. Conservative treatment includes adequate drainage, nutrition support, and somatostatin. As we now know that long-chain triglyceride (LCT) intake through the gastrointestinal tract will cause an increase in chylous leakage, ${ }^{4-7}$ nutrition support should consist of a low-fat diet supplemented with medium-chain triglyceride (MCT), an enteral nutrition (EN) plan with $\mathrm{MCT}$, or total parenteral nutrition (TPN) plan. ${ }^{4}$ Indeed, since MCT and TPN, as two nutrition support methods, can effectively prevent LCT from being absorbed by the gastrointestinal tract, they have been used to reduce chylous leakage early. ${ }^{1-4,8}$ Previous studies have compared nutrition support methods that use TPN or MCT as supplement and found that TPN was more effective than MCT. ${ }^{4,8}$ However, in those studies, MCT was used as a supplement in low-fat diets, so it still contained a small amount of LCT. EN with MCT (EN + MCT) can avoid LCT absorption by the gastrointestinal tract, and consequently reduces chylous ascites. Nevertheless, there have been hardly any reports that compare the clinical efficacy of TPN with that of EN + MCT.

Recently, the use of somatostatin to treat chyloperitoneum has been well received due to its curative effect. ${ }^{9,10}$ Octreotide, a somatostatin analog, has also been used in postoperative chylous leakage. ${ }^{11,12}$ But the use of somatostatin and octreotide in postoperative chylous ascites is mostly seen in case reports, and large-sample size studies are lacking. Therefore, the aims of our study were to evaluate the clinical effect of somatostatin and its analog octreotide in chylous ascites after abdominal surgery. Additionally, we aimed to find out whether there is a difference in the curative efficacy between plans using either TPN or EN + MCT.

\section{Materials and methods}

\section{Patients and inclusive criteria}

The study retrospectively reviewed patients who underwent abdominal surgery, from 2010 to 2014, at the West China Hospital of Sichuan University and the Affiliated Hospital of Zunyi Medical College, People's Republic of China. In this study, the abdominal surgery includes abdominal aortic aneurysm surgery, gastrointestinal tract resection, hepatopancreatobiliary surgery, cytoreduction in peritoneal metastasized disease, radical nephrectomy, retro-peritoneal lymph-node dissection, and retro-peritoneal sarcoma resection. We also excluded the specific operations-caused chylous ascites. All of the patients gave written informed consent and approved this study. The study was also approved by the Ethics Committee of Zunyi Medical College, Zunyi, People's Repbulic of China.

\section{Diagnosis of chylous ascites and trial grouping}

Diagnosis of chylous ascites was based on the noninfectious milky or creamy peritoneal fluid with a volume of $\geq 100 \mathrm{~mL} /$ day and with a triglyceride concentration of $\geq 110 \mathrm{mg} / \mathrm{dL}$. $^{13,14}$ The chylous test was used to measure chylomicrons. All patients received adequate drainage after the surgery and were given TPN from the first day after the operations. Subsequently, they were switched to either an oral diet or to an EN plan.

Once the chylous ascites was confirmed, patients received three types of nutrition supports: the MCT diet, the EN + MCT plan, and TPN plan. Type of nutrition support provided was at the discretion of the attending physicians. We use the term "EN + MCT" to refer to the EN plan with MCT, which means that the nutrition support mode contains all kinds of nutrients, but the only lipid was MCT, with no LCT.

We found that there were no significant differences in the severity of disease for patients in different groups in the preliminary analysis (data not shown). Subsequently, all of the attending physicians decisions were made according to the inclusive criteria. Therefore, we can exclude the biases among the patients in different groups.

Somatostatin was given by continuous infusion pump at a dose of $6 \mathrm{mg}$ a day. Its analog, octreotide, was given by subcutaneous injection at a dose of $0.1 \mathrm{mg}$ three times a day. The use of somatostatin or octreotide was also at the discretion of the attending doctors. So the patients could be divided into two groups: the $\mathrm{S}$ group (with somatostatin or octreotide) and the NS group (without somatostatin or octreotide).

\section{Observation of the effects of somatostatin}

The time to reach clinical success, the time to resumption of oral nutrition, the time to removal of the tube, the length of hospital stay after chylous leakage, the expenses of the chylous ascites treatments, the complications after chylous leakage, etc, were all recorded and used to evaluate the clinical effects of somatostatin. During conservative management, clinical success was defined as the disappearance of the chylous ascites. ${ }^{8}$ That required a daily drainage of milky or creamy peritoneal fluid of $<100 \mathrm{~mL} /$ day or a negative result in the chylous fluid test. 
Table I Characteristics of S group and NS group

\begin{tabular}{llll}
\hline & $\begin{array}{l}\text { S group } \\
(\mathbf{n}=\mathbf{2 8})\end{array}$ & $\begin{array}{l}\text { NS group } \\
(\mathbf{n}=\mathbf{2 2})\end{array}$ & P-value $^{\mathrm{a}}$ \\
\hline Age (years) & \\
Sex (M:F) & $60.2 \pm 10.3$ & $60.0 \pm 8.2$ & 0.934 \\
$\begin{array}{l}\text { Duration of chylous ascites } \\
\text { started (days) }\end{array}$ & $15: 13$ & $14: 8$ & $0.474^{\mathrm{c}}$ \\
$\begin{array}{l}\text { Volume of chylous } \\
\text { ascites at first day (mL) }\end{array}$ & $570.1 \pm 2.1$ & $5.8 \pm 2.3$ & 0.604 \\
$\begin{array}{l}\text { Nutrition support mode } \\
\text { (EN + MCT:TPN) }\end{array}$ & $11: 17$ & $10: 12$ & $0.661^{\mathrm{c}}$ \\
\hline
\end{tabular}

Notes: aStudent's t-test. 'Mean $\pm \mathrm{SD}$. ' Chi-square test.

Abbreviations: $M$, male; $F$, female; $E N$, enteral nutrition; $M C T$, medium-chain triglyceride; NS, without somatostatin or octreotide; TPN, total parenteral nutrition; $\mathrm{S}$ group, treatment with somatostatin or octreotide; NS group, no treatment; SD, standard deviation.

We compared the clinical efficacy of each of these strategies using MCT, EN + MCT, or TPN, with or without somatostatin. In the treatment, if the patients attained clinical success, their nutrition support would change to the MCT diet 7 days later.

\section{Statistical analysis}

Statistical analysis was performed using the SPSS software, version 19.0 (IBM Corporation, Armonk, NY, USA). The data normality test was conducted by using KolmogorovSmirnov test. Parametric data are expressed as the mean \pm $\mathrm{SD}$, and nonparametric data are expressed as the median (range). The study used the Student's $t$-test, the paired samples $t$-test, the chi-square test, and the least significant difference $t$-test to perform statistical comparisons and evaluate significance. Specific statistical analysis method will be indicated in Tables 1-3 and Figure 1. A $P$-value of $\leq 0.05$ was considered statistically significant.

\section{Results}

\section{Basic characteristics of S group and NS group patients}

There were originally 65 patients with chylous ascites complication after they underwent abdominal surgery. Two patients who received further operative treatment were excluded from the study. Five patients were also excluded because of the lack of detailed records of the curative information or the disease progress. Ultimately, 58 chylous ascites patients were included in our study. Among these patients, 36 received somatostatin (or octreotide) and were included in the S group, while the other 22, without either of these drugs, were included in the NS group. In the S group, eight patients were fed the low-fat diet supplemented with MCT as nutrition support, eleven patients were nourished with EN + $\mathrm{MCT}$, and the other 17 received TPN. In the NS group, ten patients were nourished with EN + MCT, and the other 12 received TPN as nutrition support. Hence, for comparison of the $\mathrm{S}$ and the NS groups, the eight patients fed the low-fat diet supplemented with MCT were excluded. The characteristics of these two groups are presented in Table 1.

\section{Somatostatin decreases the chylous ascites}

In the $\mathrm{S}$ group, the chylous ascites began to decrease, noticeably, 1 day after starting the treatments (Figure 1A), whereas patients in the NS group did not experience significant decrease in the chylous ascites (Figure 1B). In the course of a week's treatment, the chylous ascites volume decreased to $<100 \mathrm{~mL} /$ day in almost all the patients in the $\mathrm{S}$ group (Figure 1A), while in most patients in the NS group, it was still $>100 \mathrm{~mL} /$ day (Figure 1B).

\section{Comparison of nutrition effect for hospital stay time, treatment cost, and complications in different treatments}

The time required to attain clinical success was earlier in the S group than in the NS group (mean 4.4 \pm 2.4 vs $11.6 \pm 5.3$ days; $P<0.001)$. The tube removal time, the time to resumption of oral nutrition, and the length of hospital stay after chylous leakage of the $\mathrm{S}$ group patients were all significantly diminished compared with the NS group patients (Table 2). Some patients left the hospital with drainage tubes, so the length of hospital stay after chylous leakage in effect would be shorter than the tube removal time. The chylous ascites treatment

Table 2 Comparison of different treatments

\begin{tabular}{|c|c|c|c|}
\hline & $S$ group $(n=28)$ & NS group $(n=22)$ & $P$-value ${ }^{a}$ \\
\hline Time to clinical success (days) ${ }^{\mathrm{b}}$ & $4.4 \pm 2.4$ & $11.6 \pm 5.3$ & $<0.001$ \\
\hline Time to resume oral intake (days) ${ }^{\mathrm{b}}$ & $8.8 \pm 2.6$ & $16.4 \pm 5.6$ & $<0.001$ \\
\hline Time to tube removal (days) ${ }^{b}$ & $12.0 \pm 4.1$ & $18.6 \pm 5.8$ & $<0.001$ \\
\hline Length of hospital stay after chylous leakage (days) ${ }^{b}$ & $14.3 \pm 6.5$ & $18.3 \pm 4.9$ & 0.020 \\
\hline Expenses of chylous ascites treatment (RMB) (up to clinical success time) & $2,958.4 \pm 1,671.3$ & $3,853.4 \pm 2,3 \mid 2.3$ & 0.119 \\
\hline Complications (infection, delayed wound healing) & 1 & 6 & $0.017^{c}$ \\
\hline
\end{tabular}

Notes: 'Student's t-test. 'Mean $\pm \mathrm{SD}$. 'Chi-square test.

Abbreviations: RMB, Renminbi; S group, treatment with somatostatin or octreotide; NS group, no treatment; SD, standard deviation. 
Table 3 Comparison of different nutrition support methods in treatment with somatostatin

\begin{tabular}{|c|c|c|c|c|}
\hline & MCT $\operatorname{diet}(n=8)$ & $E N+M C T(n=I I)$ & TPN $(n=17)$ & $P$-value \\
\hline \multirow[t]{3}{*}{ Time to clinical success (days) ${ }^{\mathrm{a}}$} & $7.9 \pm 3.9$ & $4.3 \pm 2.6$ & $4.4 \pm 2.3$ & $0.009^{b}$ \\
\hline & & & & $0.006^{c}$ \\
\hline & & & & $0.94 I^{d}$ \\
\hline $\begin{array}{l}\text { Expenses of chylous ascites treatment (RMB) } \\
\text { (up to clinical success time) }\end{array}$ & No expenses & $2,|I| 1.6 \pm 1,021.4$ & $3,379.6 \pm 1,737.1$ & $0.038^{\mathrm{e}}$ \\
\hline
\end{tabular}

Notes: aMean \pm SD. ${ }^{b-d}$ One-way ANOVA (least significant difference) ('MCT diet vs EN + MCT; ' $M C T$ diet vs TPN; ${ }^{b} E N+$ MCT vs TPN). eStudent's $t$-test.

Abbreviations: MCT, medium-chain triglyceride; EN, enteral nutrition; TPN, total parenteral nutrition; RMB, Renminbi; SD, standard deviation; ANOVA, analysis of variance.

cost of the $S$ group patients was on average 2,958.4 $\pm 1,671.3$ Renminbi (RMB), whereas that of the NS group patients was 3,853.4 $\pm 2,312.3$ RMB. However, the difference was not statistically significant $(P=0.119)$. After the start of the treatments, six patients in the NS group had complications, while only one had them in the S group. Additionally, four patients in the NS group were infected at days 5, 8, 9, and 13 , respectively, and two suffered delayed wound healing as chylous fluid emerged from the wounds, whereas in the S group, only one patient was infected at day 6 . No other complications were observed in either group. Furthermore, the difference of the complications was statistically significant $(P=0.017)$.

\section{Comparison of different nutrition support methods in treatment with somatostatin}

Among the patients treated with somatostatin, the time to accomplish clinical success in patients given the MCT diet was on average $7.9 \pm 3.9$ days. On the other hand, patients fed by the TPN or EN + MCT approach demonstrated a better clinical efficacy. The time to reach clinical success in patients with the TPN plan was on average $4.4 \pm 2.3$ days, and the time to reach clinical success was $4.3 \pm 2.6$ days in patients with the EN + MCT plan. The difference among the three types of nutrition support methods was statistically significant (Table 3). Specifically, there was no statistically significant difference between the efficacy of the EN + MCT and the TPN plans $(P>0.05)$, indicating that they were basically equally effective. In contrast, the difference in efficacy between the MCT diet and the EN + MCT or TPN plans was statistically significant $(P=0.009$ and 0.008 , respectively). In patients not treated with somatostatin, the time needed to reach clinical success in patients assigned to the TPN group was on average $10.5 \pm 4.9$ days, while in patients assigned to the EN + MCT group, the time needed was $12.9 \pm 5.7$ days. The difference between these groups again was not statistically significant $(P=0.298)$. Similarly,

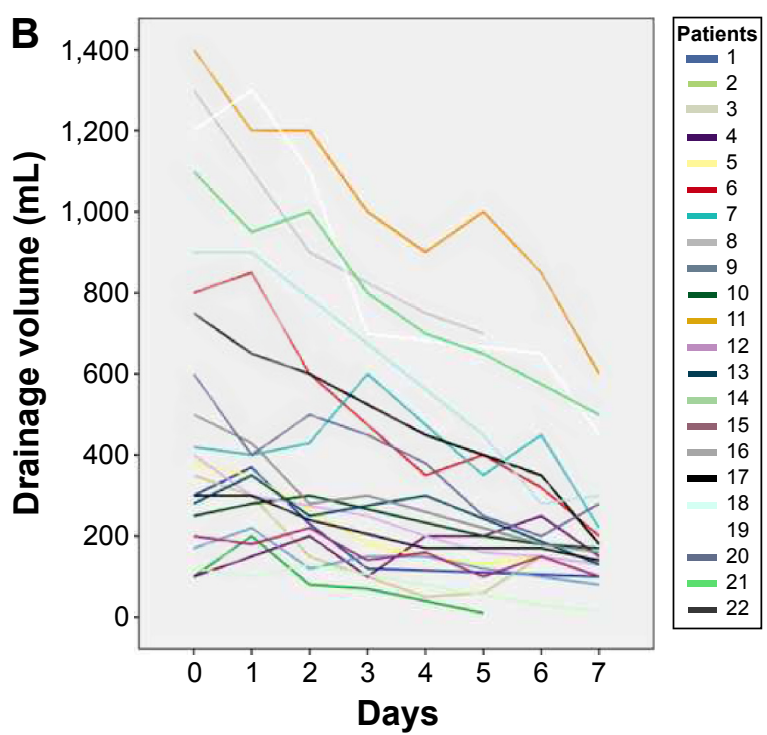

Figure I Daily changes in the volume of chylous ascites in two groups.

Notes: (A) The S group (28 patients treated with somatostatin). (B) The NS group (22 patients treated without somatostatin). The $S$ group showed significant decrease on the first day $(P<0.00 \mathrm{I})$. The NS group showed no obvious decrease $(P=0.16 \mathrm{I})$ (paired samples $t$-test).

Abbreviations: $\mathrm{S}$ group, treatment with somatostatin or octreotide; NS group, no treatment. 
the tube removal time, the time to resume oral nutrition, the length of hospital stay after chylous leakage, and the complications in patients who received the EN + MCT plan were not significantly different from those in patients on the TPN plan. Nevertheless, the cost was significantly lower $(P=0.038)$ in patients from the EN + MCT group than in those from the TPN group (mean 2,111.6 $\pm 1,021.4$

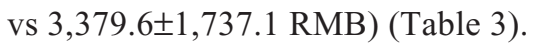

\section{Discussion}

Somatostatin and its analog octreotide inhibit the secretion of some pituitary and gastrointestinal hormones, increase splanchnic arteriolar resistance, and consequently reduce gastrointestinal flow and the lymph flow. ${ }^{15,16}$ In the study of Assumpcao et al, the mean curative time of the conservative treatment was described as 36 days. ${ }^{13}$ Kuboki et al retrospectively studied 21 patients with chylous ascites in hepatopancreatobiliary surgery and showed that the decrease of the daily drainage output (to the level of $<100 \mathrm{~mL} /$ day) was significantly earlier in patients with octreotide than in those without it (median 1 [1-2] vs 6 [1-20] days in the TPN-alone group). ${ }^{3}$ In the study of Yang et al, chylous leakage patients completely recovered within 7 days when treated with octreotide and TPN. ${ }^{17}$ The discrepancies in the conclusions of those studies were very large. Our study showed that the time needed to achieve complete clinical success was on average 4.4 days if treated with somatostatin. The tube removal time, the time to resumption of oral diet, and the length of hospital stay after chylous leakage were also much less if somatostatin was used. These results indicate that somatostatin was very effective in treating chylous ascites.

Moreover, despite the fact that somatostatin was more expensive, the cost in the two groups was not significantly different because the decrease in the therapy time by the use of somatostatin compensated for the high cost of the drug, as it decreased the incidence of complications, and decreased the cost of nutrition support and other hospital stayrelated expenses. Chylous ascites contains lymphocytes and immunoglobulins, and thus exhibits resistance to bacteria. ${ }^{1}$ However, severe and long-term chylous leakage will cause loss of a large amount of lymphatic fluid and nutrient. As a result, it will increase the incidence of infection. Out of the 50 patients who were included in this study, five were infected at about the mid-term point of the treatment. Using somatostatin decreased the drainage volume of chylous ascites significantly at the first day of the treatment. Therefore, theoretically, it would reduce the incidence of infection. In this study, four patients who were not treated with somatostatin were infected, whereas only one of those treated with somatostatin was infected. It should be noted though that the difference was not statistically significant $(P=0.087)$. Though the somatostatin, or its analog octreotide, in clinical application brought some satisfied outcomes, there were also a few controversial literatures for the somatostatin usage. ${ }^{18-21}$ Therefore, though somatostatin and its analog are helpful in preventing some of the diseases, attentive monitoring and careful intervention are also essential and critical in clinical application. ${ }^{22}$ Furthermore, the published literature has also proved that chylous ascites can also occur after thoracic surgery. ${ }^{22}$ The somatostatin was also used in this case with success. Therefore, we would perform some works to explore the mechanism of the somatostatin in the disease therapy.

The flow of the abdominal lymph fluid increases quickly after an oral diet with fat. The LCT is absorbed through lymphatic vessels of the gastrointestinal tract, while the MCT is absorbed straight into the intestinal cells and transported directly to the liver via the portal vein in the form of free fatty acids and glycerol. ${ }^{5-7}$ Therefore, MCT should be used to replace other kinds of lipids in order to decrease chylous ascites. Currently, there are three kinds of nutrition supports applied in postoperative chylous leakage treatment, namely the low-fat diet supplemented with MCT, the EN plan with MCT, and the TPN plan. ${ }^{4} \mathrm{~A}$ few studies have compared the clinical effect of MCT with TPN as a nutrition support. Tulunay et al showed that the median time to complete the clinical recovery was 28 days (range 11-60) with a low-fat diet supplemented with MCT and 10 days (range 5-66) with TPN. ${ }^{8}$ However, the low-fat diet still contains a small amount of LCT and consequently increases the volume of chylous leakage. Hence, it may be responsible for the clinical difference observed between the MCT and the TPN groups in the study of Tulunay et al. In our study, a few of the patients treated with EN + MCT completely avoided LCT absorption through the gastrointestinal tract. We compared the three types of nutrition supports which are widely used in postoperative chylous ascites: the MCT diet, the EN + MCT plan, and the TPN plan. The time that patients with the MCT diet needed to reach clinical success (mean 7.9 days) was significantly longer than that of patients on the EN + MCT plan (mean 4.3 days) or those on the TPN plan (mean 4.4 days). But there was no difference between the groups fed with EN + MCT or TPN. Just as we anticipated, as described in the "Results" section, the clinical effect of the MCT diet was of poorer quality than that of the EN + MCT and TPN plans. There was however no significant difference in the clinical efficacy between the TPN and EN + MCT plans. 
Indeed, TPN as a nutrition support method in treating postoperative chylous leakage has been amply proved to be effective, and so it was in our study. Nevertheless, there still exist obvious disadvantages of using TPN. Firstly, nutrition support with TPN usually needs long-term central venous indwelling catheter, which will increase the risk of relevant complications such as infection, thrombosis, and vascular injury. Secondly, the cost of TPN is usually very high, especially long-term use of TPN. We actually found that EN + MCT was a better nutrition support choice for postoperative chylous ascites than TPN for various reasons. First of all, the therapeutic effects had no obvious difference. Secondly, the administration of $\mathrm{EN}+\mathrm{MCT}$ does not require central venous indwelling catheter, and consequently will not have those problems associated with TPN. Finally, the cost would be significantly decreased. Therefore, nutrition support with EN + MCT was safe, effective, economical, and convenient.

The management of postoperative chylous ascites was a long-term and tough process. Our moderately large sample size study (58 patients) showed that somatostatin had obvious clinical effect, and that EN + MCT was a better nutrition support plan than the MCT diet and the TPN plan. Therefore, somatostatin in combination with EN + MCT is highly recommended in chylous ascites after abdominal surgery. Furthermore, it is plausible to generalize this result to other postoperative chylous ascites cases, including congenital and acquired chylous ascites patients.

\section{Acknowledgment}

This study was funded by a grant from the National Key Clinical Specialist Construction Programs of China (2013[544]).

\section{Disclosure}

The authors report no conflicts of interest in this work.

\section{References}

1. Aalami OO, Allen DB, Organ CH. Chylous ascites: a collective review. Surgery. 2000;128(5):761-778.

2. Leibovitch I, Mor Y, Golomb J, Ramon J. The diagnosis and management of postoperative chylous ascites. J Urol. 2002;167(2 Pt 1):449-457.

3. Kuboki S, Shimizu H, Yoshidome H, et al. Chylous ascites after hepatopancreatobiliary surgery. Br J Surg. 2013;100(4):522-527.
4. López Otero MJ, Fernández López MT, Outeiriño Blanco E. Neck chylous fistula: conservative treatment. Nutr Hosp. 2010;25(6): 1041-1044.

5. Weinstein LD, Scanlon GT, Hersh T. Chylous ascites. Management with medium-chain triglycerides and exacerbation by lymphangiography. Am J Dig Dis. 1969;14(7):500-509.

6. Ohri SK, Patel T, Desa LA, Spencer J. The management of postoperative chylous ascites. A case report and literature review. J Clin Gastroenterol. 1990;12(6):693-697.

7. Cárdenas A, Chopra S. Chylous ascites. Am J Gastroenterol. 2002; 97(8):1896-1900.

8. Tulunay G, Ureyen I, Turan T, Karalok A. Chylous ascites: analysis of 24 patients. Gynecol Oncol. 2012;127(1):191-197.

9. Karaca S, Gemayel G, Kalangos A. Somatostatin treatment of a persistent chyloperitoneum following abdominal aortic surgery. J Vasc Surg. 2012;56(5):1409-1412.

10. Giovannini I, Giuliante F, Chiarla C, et al. External lymphatic fistula after intra-abdominal lymphadenectomy for cancer. Treatment with total parenteral nutrition and somatostatin. Nutrition. 2008;24(11-12): $1220-1223$.

11. Pessotti CF, Jatene IB, Buononato PE, Elias PF, Pinto AC, Kok MF. Use of octreotide in the treatment of chylothorax and chyloperitoneum. Arq Bras Cardiol. 2011;97(2):e33-e36.

12. Kim EA, Park H, Jeong SG, Lee C, Lee JM, Park CT. Octreotide therapy for the management of refractory chylous ascites after a staging operation for endometrial adenocarcinoma. J Obstet Gynaecol Res. 2014;40(2):622-626.

13. Assumpcao L, Cameron JL, Wolfgang CL, et al. Incidence and management of chyle leaks following pancreatic resection: a high volume single-center institutional experience. J Gastrointest Surg. 2008;12(11): 1915-1923.

14. Aoki H, Takakura N, Shiozaki S, Matsukawa H. Milk-based test as a preventive method for chylous ascites following pancreatic resection. Dig Surg. 2010;27(5):427-432.

15. Cheung Y, Leung MP, Yip M. Octreotide for treatment of postoperative chylothorax. J Pediatr. 2001;139(1):157-159.

16. Berzigotti A, Magalotti D, Cocci C, Angeloni L, Pironi L, Zoli M. Octreotide in the outpatient therapy of cirrhotic chylous ascites: a case report. Dig Liver Dis. 2006;38(2):138-142.

17. Yang C, Zhang J, Wang S, Li CC, Kong XR, Zhao Z. Successful management of chylous ascites with total parenteral nutrition and octreotide in children. Nutr Hosp. 2013;28(6):2124-2127.

18. Guo L, Tang CW. Somatostatin analogues do not prevent carcinoid crisis. Asian Pac J Cancer Prev. 2014;15(16):6679-6683.

19. Gurusamy KS, Koti R, Fusai G, Davidson BR. Somatostatin analogues for pancreatic surgery. Cochrane Database Syst Rev. 2013;4:CD008370.

20. Horvers M, Mooij CF, Antonius TA. Is octreotide treatment useful in patients with congenital chylothorax? Neonatology. 2012;101(3): 225-231.

21. Das A, Shah PS. Octreotide for the treatment of chylothorax in neonates. Cochrane Database Syst Rev. 2010;9:CD006388.

22. Karaca S, Rager O, Kalangos A. Successful treatment of persistent chylopericardium with somatostatin after operation on ascending aorta. Ann Thorac Surg. 2014;97(4):e97-e99.
Therapeutics and Clinical Risk Management

\section{Publish your work in this journal}

Therapeutics and Clinical Risk Management is an international, peerreviewed journal of clinical therapeutics and risk management, focusing on concise rapid reporting of clinical studies in all therapeutic areas, outcomes, safety, and programs for the effective, safe, and sustained use of medicines. This journal is indexed on PubMed Central, CAS,

\section{Dovepress}

EMBase, Scopus and the Elsevier Bibliographic databases. The manuscript management system is completely online and includes a very quick and fair peer-review system, which is all easy to use. Visit $\mathrm{http}: / /$ www.dovepress.com/testimonials.php to read real quotes from published authors. 